\title{
Polymer self-locking clips for vascular control during minimally invasive pulmonary lobectomies
}

\author{
Marco Lucchi, MD, Leonardo Duranti, MD, Franca Melfi, MD, and Alfredo Mussi, MD, Pisa, Italy
}

Supplemental material is available online.

Control and division of the hilar vessels is a crucial step in performing minimally invasive pulmonary lobectomy safely and efficiently.

Hem-o-lok clips (Teleflex Medical, Inc, Reading, Pa) were first introduced in 1999 and have already been used and validated for laparoscopic nephrectomy, ${ }^{1}$ as well as other urologic and general surgery procedures. They are nonabsorbable polymer clips characterized by a lock-engagement feature, which ensures the closure of the clip itself, and teeth within the jaws, designed to prevent slippage. Hem-o-lok clips come in 4 sizes. Their curved shape helps the surgeon to surround the vessels. Three applicator tools have been developed: one for open surgery, one for endoscopic surgery, and another for robotic surgery. Applicators for open surgery come in 2 forms: angular or straight. The endoscopic device is only straight, whereas the robotic device is assembled on the robotic EndoWrist instrument (Intuitive Surgical, Inc, Mountain View, Calif) and has 7 degrees of mobility.

To test this surgical device on pulmonary artery branches, we performed a prospective trial on consecutive cases of pulmonary lobectomies performed through a minithoracotomy.

\section{PATIENTS AND METHODS}

From February 2007 to February 2008, all patients with clinical stage I non-small cell lung cancer or low-grade neoplasms who were in suitable condition for a pulmonary lobectomy through a minithoracotomy, and who gave their written informed consent, were enrolled in our study.

\footnotetext{
From the Division of Thoracic Surgery, Cardiac and Thoracic Department, University of Pisa, Pisa, Italy.

Disclosures: The authors received no financial support from the related company. All items mentioned in this article were purchased by our institution on the basis of the market.

Received for publication Sept 9, 2009; revisions received Oct 24, 2009; accepted for publication Dec 12, 2009; available ahead of print Feb 22, 2010.

Address for reprints: Marco Lucchi, MD, Division of Thoracic Surgery, Cardiac and Thoracic Department, University of Pisa, Via Paradisa 2, Pisa 56124, Italy (E-mail: m.lucchi@med.unipi.it).

J Thorac Cardiovasc Surg 2010;139:1345-6

$0022-5223 / \$ 36.00$

Copyright $(2010$ by The American Association for Thoracic Surgery

doi:10.1016/j.jtcvs.2009.12.026
}

All procedures were performed by vertical axillary minithoracotomy or small anterior thoracotomy. For each patient, relevant preoperative clinical data, the number and size of clips used, the operative time, estimated blood loss, early and late complications, and length of hospital stay were analyzed.

Exclusion criteria were as follows: tumor size greater than $5 \mathrm{~cm}$ with hilar extension and hilar or mediastinal lymph node involvement on the preoperative computed tomogram.

Once pulmonary vessels were accurately dissected, the lobar arteries were controlled separately by correct placement of the Hem-o-lok clips. The lobar vessels must be thoroughly skeletonized to allow viewing of the Hem-o-lok tips clipping together and thereby ensure that the clips are closed (Figure E1). Two locking clips were applied on the hilar side and another one was positioned on the pulmonary side to guarantee safety in this process. Subsequently, the hilar vessels were transected by scissors, leaving at least a 1-mm cuff adjacent to the clips (Figure E2).

\section{RESULTS}

A total of 47 patients (27 men and 20 women) were included in this study. We performed the following lobectomies: 12 right upper, 5 middle lobe, 11 right lower, 10 left upper, and 9 left lower. The average operative time was $107 \pm 28$ minutes, and the average operative blood loss was $150 \pm 30 \mathrm{~mL}$. Overall, a total of 109 pulmonary artery branches were encountered. In 107 we used Hem-o-lok clips $(98.2 \%)$. In 2 cases the artery branch was not sufficiently dissected because of the presence of enlarged hilar lymph nodes; as a consequence, the vessel was not long enough to place 3 clips, and we considered a running 4-0 Prolene polypropylene suture (Ethicon, Inc, Somerville, NJ) to be safer. It was not the purpose of our study to use the Hemo-lok clips for venous ligation; however, in 9 cases we successfully tested the clips also for the vein (Figure E1).

To select the appropriate size of the clips to be used, we followed the protocol shown in Table 1 .

Overall, we did not observe any direct vascular damage, clip failure (reopening), or slippage, nor did we experience any early or late complication related to the use of the clips.

\section{CONCLUSIONS}

Vascular control in a video-assisted and robot-assisted setting is usually achieved by endostaplers, ${ }^{2}$ the application of which on a main branch of the pulmonary artery always determines some degree of tension. Traction along the 
TABLE 1. Size of the clips suitable for pulmonary lobectomies

\begin{tabular}{llll}
\hline \multicolumn{1}{c}{ Right side lobectomies } & & Left side lobectomies \\
\hline Upper lobectomy & & & Large clips \\
Truncus anterior & Large clips & Truncus anterior & Medium-large clips \\
$\begin{array}{l}\text { Ascending (posterior/anterior) } \\
\text { Middle lobectomy }\end{array}$ & Medium-large clips & Ascending (posterior/anterior) & \\
$\begin{array}{l}\text { Single artery }(46 \%) \\
\text { Double artery (51\%) }\end{array}$ & Medium-large clips & \\
Lower lobectomy & Medium-large clips & & \\
Apical segmental & & Apical segmental & Medium-large clips \\
Basal trunk & Medium-large clips & Basal trunk & Large clips \\
\hline
\end{tabular}

branch could even result in its avulsion. ${ }^{3}$ Another risk is the failing alignment of the two jaws of the stapler, leading to incorrect positioning of the staples. Primary mechanical malfunction is also possible, ${ }^{3,4}$ and the placement of the stapler by the assistant is not easy during robotically assisted lobectomy. ${ }^{5}$ Last but not least, staplers are expensive (endovascular stapler +1 cartridge $=479$ euros in our hospital) compared with the cost of the Hem-o-lok clips (6-clip blister pack $=46$ euros), and the applicator is free and reusable.

Considering the use of Hem-o-lok clips in pulmonary surgery, we found some favorable conditions. First, the pressure of the pulmonary artery is lower than that of the systemic arteries and the dissection of the lobar branches usually allows the surgeon to achieve a vessel length sufficient to place 3 clips and maintain a 1- to 2-mm cuff of vessel, which is highly recommended. Moreover, it is important to select the appropriate size of the clips to be used; the clips cannot be smaller than the vessel, but should not be larger.

In conclusion, our preliminary experience suggests the utility of Hem-o-lok clips in minimally invasive pulmonary lobectomies. Further larger studies about reliability, safety, and cost-effectiveness are warranted.

\section{References}

1. Ponsky L, Cherullo E, Moinzadeh A, Desai M, Kaouk J, et al. The Hem-o-lok clip is safe for laparoscopic nephrectomy: a multi-institutional review. Urology. 2008; 71:593-6.

2. Szwerc MF, Landreneau RJ, Santos RS, Keenan RJ, Murray GF. Minithoracotomy combined with mechanically stapled bronchial and vascular ligation for anatomical lung resection. Ann Thorac Surg. 2004;77:1904-9; discussion 1909-10.

3. Craig SR, Walker WS. Potential complications of vascular stapling in thoracoscopic pulmonary resection. Ann Thorac Surg. 1995;59:736-7; discussion 737-8.

4. Yim AP, Ho JK. Malfunctioning of vascular staple cutter during thoracoscopic lobectomy. J Thorac Cardiovasc Surg. 1995;109:1252.

5. Melfi FM, Mussi A. Robotically assisted lobectomy: learning curve and complications. Thorac Surg Clin. 2008;18:289-95.

\title{
The option of taking down the Fontan circulation: The Melbourne experience
}

\author{
Ajay J. Iyengar, MBBS, BMedSci, Christian P. Brizard, MD, MS, Igor E. Konstantinov, MD, PhD, and \\ Yves d' Udekem, MD, PhD, Melbourne, Australia
}

\footnotetext{
From the Department of Cardiac Surgery, Royal Children's Hospital, the University of Melbourne Department of Paediatrics, and the Murdoch Children's Research Institute, Melbourne, Australia.

Disclosures: None.

Received for publication Sept 16, 2009; revisions received Nov 20, 2009; accepted for publication Dec 13, 2009; available ahead of print March 10, 2010.

Address for reprints: Yves d'Udekem, MD, PhD, Department of Cardiac Surgery, Royal Children's Hospital, Flemington Road, Parkville, Melbourne 3052, Victoria, Australia (E-mail: yves.dudekem@rch.org.au).

J Thorac Cardiovasc Surg 2010;139:1346-8 0022-5223/\$36.00

Crown Copyright $\Subset 2010$ Published by Elsevier Inc. on behalf of The American Association for Thoracic Surgery

doi:10.1016/j.jtcvs.2009.12.029
}

In recent years, dramatic early failure of the Fontan circulation has become exceedingly rare, ${ }^{1}$ although there are occasional deaths in patients with large ongoing chest drain losses. Fontan takedown to a prior palliative state has been described to allow successful survival of these patients, but only limited information exists on the early and longterm results of this rescue procedure. ${ }^{2}$

\section{CLINICAL SUMMARY}

Between January 1980 and December 2007, 413 patients underwent a Fontan procedure at the Royal Children's Hospital, Melbourne. During this period, 14 

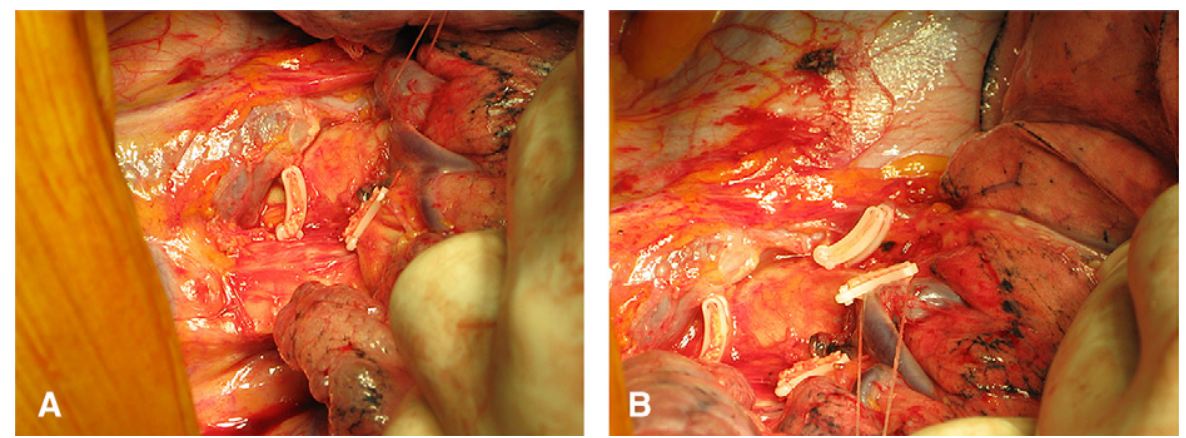

FIGURE E1. The truncus anterior arterial branch $(A)$ and upper lobe vein $(B)$ sutured by large clips during a right upper lobectomy.
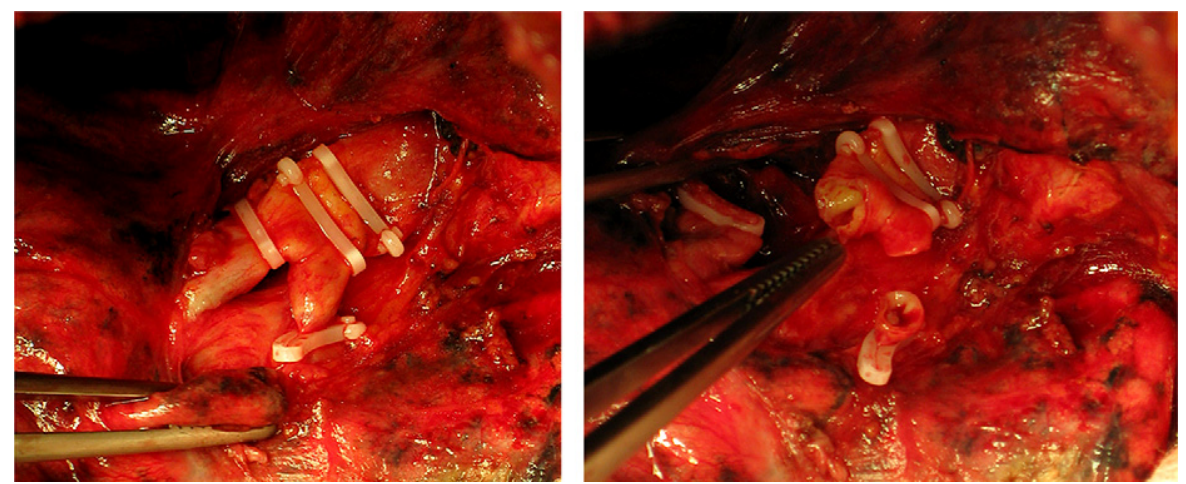

FIGURE E2. Large and medium-large clips positioned during a left lower lobectomy. 